\title{
ANTIBIOGRAM OF ENTEROCOCCAL SPECIES ISOLATED FROM CLINICAL SPECIMENS IN A TERTIARY CARE TEACHING HOSPITAL
}

\author{
Mohan Sundaram ${ }^{1}$, Yedla Kavita², Shaik Khaja Mohiddin ${ }^{3}$ \\ ${ }^{1}$ Assistant Professor, Department of Microbiology, Vinayaka Missions Medical College, Karaikal. \\ ${ }^{2}$ Assistant Professor, Department of Microbiology, Vinayaka Missions Medical College, Karaikal. \\ ${ }^{3}$ Tutor, Department of Microbiology, Vinayaka Missions Medical College, Karaikal.
}

ABSTRACT
BACKGROUND
Enterococci are Gram-positive cocci that are normal inhabitants of the gastrointestinal tract. Enterococci have become the second
most common agent recovered from nosocomial UTI and wound infections and the third leading cause of bacteraemia. Although,
enterococci have been considered of relatively low virulence, these organisms can cause serious infections including endocarditis.
In the last few decades, the number of serious infections caused by these organisms has been steadily increasing. Resistance to
several commonly used antibiotics is a remarkable characteristic of most of the enterococcal species. We aimed at determining the
isolation rate and resistance pattern of Enterococcal species to different antibiotics from clinical specimens.

MATERIAL AND METHODS

A total of 107 Enterococcal species were isolated and identified from different clinical samples by standard microbiological tests. Antimicrobial susceptibility testing was performed by Kirby-Bauer's disc diffusion method as per CLSI guidelines.

\section{RESULTS}

Enterococcus faecalis 85 (79.44\%) were predominantly isolated; 22 strains of Enterococcus faecium were isolated and accounted for $20.56 \%$. Majority of isolates were from urine 87 (81.31\%) followed by pus 14 (13.08\%). High degree of resistance was observed towards penicillin and ampicillin accounting for $94.39 \%$. High level of drug resistance was observed towards gentamicin (64.49\%) followed by streptomycin (58.88\%). All isolates were found to be susceptible to linezolid and vancomycin.

\section{CONCLUSION}

Enterococcus faecium is comparatively more resistant than Enterococcus faecalis. Identification of Enterococci up to species level may help the clinician to choose the appropriate therapy. Antimicrobial surveillance should be done periodically to monitor the current susceptibility patterns in local hospitals.

\section{KEYWORDS}

Enterococci, Uropathogen, Antibiogram, Vancomycin, Linezolid.

HOW TO CITE THIS ARTICLE: Sundaram M, Kavita Y, Mohiddin SK. Antibiogram of enterococcal species isolated from clinical specimens in a tertiary care teaching hospital. J. Evolution Med. Dent. Sci. 2016;5(47):2955-2958, D0I: 10.14260/jemds/2016/689

\section{INTRODUCTION}

Enterococci are primarily members of the gastrointestinal microflora of humans that act as opportunistic pathogens. ${ }^{1}$ Gastrointestinal tract is the site, which is believed to be the reservoir for strains associated with disease. From gastrointestinal tract, Enterococci may migrate to cause infections and also can disseminate to other hosts and environmental surfaces. ${ }^{2}$ But in the last few decades, the number of serious infections caused by these organisms has been steadily increasing. Enterococci have become the second most common agent recovered from nosocomial UTI and wound infections and the third leading cause of bacteremia. ${ }^{3}$ Intra-abdominal and intra-pelvic infections are the next most commonly encountered infections. However, cultures from patients with peritonitis, intra-abdominal, biliary tract

Financial or Other, Competing Interest: None.

Submission 27-04-2016, Peer Review 21-05-2016,

Acceptance 27-05-2016, Published 10-06-2016.

Corresponding Author:

Mohan Sundaram,

Vinayaka Missions Medical College,

Karaiakal-609609,

Puducherry.

E-mail:drmohanmmc@gmail.com

DOI: $10.14260 /$ jemds/2016/689 infections and endomyometritis are frequently polymicrobial and the role of Enterococci in this setting is controversial. ${ }^{4}$

In humans enterococcal infections may be caused by at least 12 species, but most clinical infections are due to either Enterococcus faecalis or E. faecium. E. faecalis is the most common cause $(80-90 \%)$ followed by E. faecium (10-15\%). Occasional infections are due to Enterococcus gallinarum, Enterococcus raffinosus, Enterococcus casseliflavus, Enterococcus avium, Enterococcus pseudoavium, Enterococcus malodoratus, Enterococcus mundtii, Enterococcus durans and Enterococcus hirae. ${ }^{5}$

Antibiotic resistance among Enterococci is a major obstacle for treatment. The relative importance of Enterococcus as a pathogen has increased with the occurrence of high-level resistance to multiple antimicrobial drugs such as ampicillin, aminoglycosides and vancomycin. ${ }^{6}$ Ongoing surveillance of Enterococcal resistance against antimicrobial agents is fundamental to monitor trends in susceptibility patterns and to appropriately guide the clinician in choosing empirical or directed therapy.

Hence, we aimed at determining the isolation rate and resistance pattern of Enterococci to different antibiotics from clinical specimens. 


\section{MATERIALS AND METHODS}

This is a prospective, observational study in which a total of 107 Enterococci were isolated from clinical specimens received in the Department of Clinical Microbiology over a period of eighteen months. The clinical significance of the Enterococcal species was assessed retrospectively by analysing the case sheets for compiling of laboratory and clinical criteria. The isolates were identified based on colony characters, morphology on gram staining and biochemical reactions using conventional test scheme by Facklam et al. ${ }^{7}$ Identification of Enterococci isolates was confirmed on the basis of the growth of these organisms on bile-esculin medium, presence of gram-positive cocci in pairs and short chains on gram staining of these colonies, catalase-negative colonies and growth of these organisms in $6.5 \% \mathrm{NaCl}$ and at $\mathrm{pH} 9.6$. Enterococcal strains were further identified to the species level by using conventional physiological tests, which are based on carbohydrate fermentation using $1 \%$ solution of the following sugars: glucose, mannitol, arabinose, raffinose, sorbitol, sucrose, lactose, trehalose and inulin; by pyruvate utilization in $1 \%$ pyruvate broth; arginine decarboxylation in Moeller's decarboxylase broth; hippurate hydrolysis; motility test; pigment production detected on Tryptic Soy Agar (TSA); gelatin liquefaction; starch hydrolysis using $2 \%$ starch and polysaccharide production. A single colony isolate was inoculated into $5 \mathrm{~mL}$ Todd-Hewitt broth and incubated overnight at $37^{\circ} \mathrm{C}$, which was then added as an inoculum of one drop with the help of Pasteur pipette. All tests were incubated at $37^{\circ} \mathrm{C}$ and read at 24 hours and 7 days.

\section{RESISTANCE PROFILE}

\section{Antibiotic Susceptibility Pattern}

Antimicrobial susceptibility test was performed by KirbyBauer disc diffusion method. The peptone water culture standardized to 0.5 McFarland opacity was used for surface seeding on Mueller Hinton agar. After plates were dried, antibiotic discs were placed over the medium and incubated at $37^{\circ} \mathrm{C}$ for 24 hours. ${ }^{8}$ Following antibiotics were used to determine the susceptibility pattern of all enterococcal strains. Ciprofloxacin (CIP) (5 $\mu \mathrm{g})$, ampicillin (AMP) $(10 \mu \mathrm{g})$, penicillin (P) $(10 \mathrm{IU})$, gentamicin (HLG) $(120 \mu \mathrm{g})$, streptomycin (HLS) $(300 \mu \mathrm{g})$, Linezolid $(\mathrm{Le}-30 \mu \mathrm{g})$, Vancomycin (Va-30 $\mu \mathrm{g})$.

E. faecalis ATCC 29212 was used as a quality control strain for performing antimicrobial tests.

\section{RESULTS}

During the study period, a total of 107 Enterococci were isolated. Enterococcus faecalis 85 (79.44\%) were predominantly isolated; 22 strains of Enterococcus faecium were isolated and accounted for $20.56 \%$. No other species were isolated. Majority of isolates were from urine 87 (81.31\%) followed by pus 14 (13.08\%), blood $4(3.74 \%)$ and body fluids $2(1.87 \%)$ [Table 1$]$.

\begin{tabular}{|c|c|c|c|}
\hline $\begin{array}{c}\text { Clinical } \\
\text { Specimen }\end{array}$ & $\begin{array}{c}\text { E. } \\
\text { Faecalis }\end{array}$ & $\begin{array}{c}\text { E. } \\
\text { Faecium }\end{array}$ & Total \\
\hline Urine & 71 & 16 & $\begin{array}{c}87 \\
(81.31 \%)\end{array}$ \\
\hline Pus & 9 & 5 & $\begin{array}{c}14 \\
(13.08 \%)\end{array}$ \\
\hline Blood & 3 & 1 & $4(3.74 \%)$ \\
\hline Body Fluids & 2 & 0 & $2(1.87 \%)$ \\
\hline \multicolumn{3}{|c|}{ Table 1: Specimen Wise Distribution } \\
of E. faecalis and E. faecium \\
\hline
\end{tabular}

Majority of the isolates exhibited high degree of resistance towards penicillin and ampicillin accounting for $94.39 \%$. High level of drug resistance was observed towards gentamicin $(64.49 \%)$ followed by streptomycin (58.88\%). Majority of E. faecium isolates showed resistance to HLG (High Level Gentamicin) and HLS (High Level Streptomycin). All isolates were found to be susceptible to teicoplanin and vancomycin. [Table 2].

\begin{tabular}{|c|c|c|c|}
\hline Antibiotic & $\begin{array}{c}\text { E. faecalis } \\
(n=85)\end{array}$ & $\begin{array}{c}\text { E. faecium } \\
(n=22)\end{array}$ & $\begin{array}{l}\text { Total } \\
(107) \\
\end{array}$ \\
\hline Penicillin & 85 (100\%) & $22(100 \%)$ & $\begin{array}{c}107 \\
(100 \%) \\
\end{array}$ \\
\hline Ampicillin & $\begin{array}{c}79 \\
(92.94 \%) \\
\end{array}$ & $22(100 \%)$ & $\begin{array}{c}101 \\
(94.39 \%) \\
\end{array}$ \\
\hline $\begin{array}{c}\text { Gentamicin } \\
\text { (HLG) }\end{array}$ & $\begin{array}{c}52 \\
(61.18 \%) \\
\end{array}$ & $\begin{array}{c}17 \\
(77.27 \%) \\
\end{array}$ & $\begin{array}{c}69 \\
(64.49 \%) \\
\end{array}$ \\
\hline $\begin{array}{c}\text { Streptomycin } \\
\text { (HLS) }\end{array}$ & $\begin{array}{c}49 \\
(57.65 \%) \\
\end{array}$ & $\begin{array}{c}14 \\
(63.64 \%) \\
\end{array}$ & $\begin{array}{c}63 \\
(58.88 \%) \\
\end{array}$ \\
\hline Ciprofloxacin & $\begin{array}{c}42 \\
(49.41 \%)\end{array}$ & $11(50 \%)$ & $\begin{array}{c}53 \\
(49.53 \%)\end{array}$ \\
\hline Linezolid & $0(0 \%)$ & $0(0 \%)$ & $0(0 \%)$ \\
\hline Vancomycin & $0(0 \%)$ & $0(0 \%)$ & $0(0 \%)$ \\
\hline
\end{tabular}

\section{DISCUSSION}

Resistance to several commonly used antibiotics is a remarkable characteristic of most of the enterococcal species. Moreover, majority of information available is based on studies with Enterococcus faecalis and Enterococcus faecium, the two species that are more commonly involved in causing human infections. In our study, majority of isolates were isolated from urine $(81.31 \%)$ followed by pus $(13.08 \%)$. In other studies also, urine was the most common sample yielding enterococci; Mathur et al $^{9}$ obtained $49 \%$, Karmarkar et al ${ }^{10}$ obtained $50 \%$ and Udo et al ${ }^{11}$ obtained $36.6 \%$ of enterococci from urine samples. However, few studies reported predominant isolation rate of Enterococci from pus followed by urine. ${ }^{12}$ Enterococcus species were found to be predominantly isolated from in-patient departments, which was associated with the patient's critical illness, long-term antibiotic use and decline in immune function.

In our study, Enterococcus faecalis (79.44\%) was the predominant isolate followed by Enterococcus faecium (20.56\%). No other enterococcal species were isolated in our study. This is in agreement with the previous studies conducted by Karmarkar et $\mathrm{al}^{10}$ and Mendiratta et al. ${ }^{13}$ However, recent studies have shown an increase in the isolation rate of Enterococcus faecium and other non-faecalis species of Enterococcus. ${ }^{14}$ Karmarkar et al ${ }^{10}$ from Mumbai reported higher isolation of Enterococcus faecium (80.7\%) over Enterococcus faecalis (19.2\%) in their study, which is not in agreement with the present study.

Enterococci are intrinsically resistant to many antibiotics. Unlike acquired resistance and virulence traits, which are usually transposon or plasmid encoded, intrinsic resistance is based on chromosomal genes, which typically are nontransferrable. The frequency of penicillin and ampicillin resistance was high in the present study. Reports of the steady rise in the recovery rates of Ampicillin-Resistant Enterococci (ARE) have been available in the recent past in India. 15 
Among quinolones, least sensitivity was observed with ciprofloxacin. Similar low sensitivity with ciprofloxacin was reported by Subbalaxami et al. ${ }^{16}$

High-Level Resistance to Aminoglycosides (HLAR) is of great clinical concern since it eliminates synergy with cell wall active antibiotics, which renders treatment of serious enterococcal infections difficult. 17 Enterococci show intrinsic low-level cross resistance to all aminoglycosides due to decreased uptake of antibiotics. Therefore, there is no meaning in testing susceptibility of clinical isolates of enterococci to low-level aminoglycosides. Acquired resistance to high level of aminoglycosides can also be present in enterococci due to genes encoding Aminoglycoside Modifying Enzymes (AMEs). Aminoglycosides are frequently used in combination with cellwall-active antibiotics for severe enterococcal infections. 18 Resistance mechanisms of enterococci to gentamicin and to streptomycin differs. Hence, it is necessary to perform susceptibility to both agents. Enterococci with high level resistance to streptomycin are susceptible to gentamicin. Gentamicin resistance is a good predictor of resistance to other aminoglycosides except streptomycin. ${ }^{5}$ Out of 107 enterococcal isolates, $64.49 \%$ were found to be HLGR and $58.88 \%$ were HLSR. Various studies have also indicated HLGR to be more common than HLSR in all species of enterococci. ${ }^{19}$ Both HLGR (High Level Gentamicin Resistance) and HLSR (High Level Streptomycin Resistance) were seen to be more common in E. faecium as compared to E. faecalis. These results are in concurrence with the results of other studies. ${ }^{9}$

With the spread of strains showing HLAR (High Level Aminoglycoside Resistance), there is now rampant use of vancomycin in hospitals since it is the only available alternative for treatment. Based on our findings, good antienterococcal activity was observed in $100 \%$ with both linezolid and vancomycin. Probably, this is due to less usage of these antibiotics in this region. Various studies from India reported vancomycin resistance in a range of $1.7-20 \%{ }^{20}$ However, in a study conducted by Deshpande et al,17 less than $2 \%$ of E. faecalis were found to be resistant to vancomycin, whereas $52 \%$ of the E. faecium isolates were resistant to vancomycin. The frequency and extent of glycopeptides resistance in a study conducted by Deshpande et al,17 were much higher compared to those of previous reports from India. 21

It is indicated that the resistance to glycopeptides in enterococci is mainly caused by the alteration of peptidoglycan precursors on the cell wall of enterococci, which leads to the failure of the glycopeptides to inhibiting the synthesis of the cell walls of enterococci, thereby resulting in the emergence of glycopeptide resistance. ${ }^{22}$ The acquisition of vancomycin resistance by enterococci has seriously affected the treatment and infection control of these organisms. VRE, particularly E. faecium strains, are frequently resistant to all antibiotics that are effective treatment for vancomycin-susceptible enterococci, which leaves clinicians treating VRE infections with limited therapeutic options.

Newer antibiotics (e.g., quinupristin-dalfopristin, linezolid, daptomycin, tigecycline) with activity against many VRE strains have improved this situation, but resistance to these agents has already been described. A mutation (G2576U) in the domain $\mathrm{V}$ of the $23 \mathrm{~S}$ rRNA is responsible for linezolid resistance. ${ }^{17}$
Whereas resistance to quinupristin-dalfopristin may be the result of several mechanisms: modification of enzymes, active efflux and target modification. Resistance of E. faecalis and E. faecium to daptomycin, a newer cyclic lipopeptide antibiotic that acts on the bacterial cell membrane has also been reported. 23

Overall, in our study E. faecium is comparatively more resistant than E. faecalis. Many studies have also demonstrated that E. faecium is more resistant than E. faecalis. The monitoring of the prevalence and antimicrobial resistance of Enterococcus species would provide a guide for the appropriate selection of antibiotics and prevent the occurrence of more antimicrobial-resistant enterococcal isolates. The problem of treatment and control of enterococcal infections is underscored by the high prevalence of nosocomial isolates and their ability to acquire resistance to the limited number of useful antimicrobial agents available in the treatment of enterococcal infections. The results of our study are based on phenotypic methods alone. No minimum inhibitory concentration technique was used to detect Vancomycin Resistant Enterococci (VRE), which remained as limitations of our study.

\section{CONCLUSION}

Enterococcal species have great potential to survive in hospital environment. So improved antibiotic stewardship and infection? ?control measures will be needed to prevent or slow the emergence and spread of multidrug? resistant Enterococci in the healthcare setting. A combination of control measures was implemented to contain these organisms in our setup. Prudent use of vancomycin and a proper surveillance for Vancomycin resistant Enterococci may permit early recognition and containment of spread of this emerging pathogen in our country. Enterococcus faecium is comparatively more resistant than Enterococcus faecalis. Identification of Enterococci up to species level may help the clinician to choose the appropriate therapy.

\section{REFERENCES}

1. Kaye D. Enterococci biologic and epidemiologic characteristics and in vitro susceptibility. Arch Intern Med 1982;142(11):2006-9.

2. Murray BE, Singh KV, Markowitz SM, et al. Evidence for clonal spread of a single strain of $ß$-lactamase-producing enterococcus (streptococcus) faecalis to six hospitals in five states. J Infect Dis 1991;163(4):780-5.

3. Schaberg DR, Culver DH, Gaynes RP. Major trends in the microbial aetiology of nosocomial infection. American Journal of Medicine 1991;91(3B):S72-5.

4. Gilmore MS, Segarra RA, Booth MC. An hlyB-type function is required for expression of the enterococcus faecalis hemolysin/bacteriocin. Infect Immun 1990;58(12):391423.

5. Sood S, Das MM, Kapil A. Enterococcal infections and antimicrobial resistance. Indian J Med Res 2008;128:11121.

6. Marothi YA, Agnihotri H, Dubey D. Enterococcal resistance-an overview. Indian J Med Microbiol 2005;23(4):214-9.

7. Facklam RR, Collins MD. Identification of enterococcus species isolated from human infections by a conventional test scheme. J Clin Microbiol 1989;27(4):731-4. 
8. Clinical and laboratory standards institute. Performance standards for antimicrobial susceptibility testing, informational supplement (M100-S17), 17th edn. Clinical and Laboratory Standards, Wayne 2007;27(1).

9. Mathur P, Kapil A, Chandra R, et al. Antimicrobial resistance in enterococcus faecalis at a tertiary care centre in northern India. Indian J Med Res 2003;118:25-8.

10. Karmarkar MG, Gershom ES, Mehta PR. Enterococcal infections with special reference to phenotypic characterization \& drug resistance. Indian J Med Res 2004;119:22-5.

11. Udo EE, Al-Sweih N, Philips OA, et al. Species prevalence and antibacterial resistance of enterococci isolated in Kuwait hospitals. J Med Microbiol 2003;52(2):163-8.

12. Vandamme P, Vercauteran E, Lammens C, et al. Survey of enterococcal susceptibility pattern in Belgium. J Clin Microbiol 1996;34(10):2572-6.

13. Mendiratta DK, Kaur H, Deotale V, et al. Status of high level aminoglycoside resistant enterococcus faecium and enterococcus faecalis in a rural hospital of central India. Indian J Med Microbiol 2008;26(4):369-71.

14. Jain S, Kumar A, Kashyap B, et al. The clinicoepidemiological profile and the high-level aminoglycoside resistance in enterococcal septicemia at a tertiary care hospital in east Delhi. Int J App Basic Med Res 2011;1(2):80-3.

15. Sekar R, Srivani R, Vignesh R, et al. Low recovery rates of high-level aminoglycoside-resistant enterococci could be attributable to restricted usage of aminoglycosides in Indian settings. J Med Microbiol 2008;57(3):397-8.
16. Subbalaxmi MVS, Lakshmi V, Lavanya V. Antibiotic resistance experience in a tertiary care hospital in south India. Journal of Association of Physicians of India 2010;58:18-22.

17. Deshpande VR, Karmarkar MG, Mehta PR. Prevalence of multidrug resistant enterococci in a tertiary care hospital in Mumbai, India. J Infect Dev Ctries 2013;7(2):155-8.

18. Arias CA, Murray BE. Emergence and management of drug-resistant enterococcal infections. Expert Rev Anti Infect Ther 2008;6(5):637-55.

19. Padmasini E, Padmaraj R, Ramesh SS. High level aminoglycoside resistance and distribution of aminoglycoside resistant genes among clinical isolates of enterococcus species in Chennai, India. Sci World J 2014;2014:5.

20. Vidyalakshmi PR, Gopalakrishnan R, Ramasubramanian V, et al. The clinical, epidemiological and the microbiological profiles of the patients with vancomycin-resistant enterococci from a tertiary care hospital. J Global Infect Dis 2012;4(2):137-8.

21. Shafiyabi S, Mariraj J, Sumathi S, et al. Emergence of vancomycin resistant enterococci in a tertiary care hospital in south India. Int J Pharm Biomed Res 2013;4(2):111-3.

22. Arthur M, Reynolds P, Courvalin P. Glycopeptide resistance in enterococci. Trends Microbiol 1996;4(10):401-7.

23. Long JK, Choueiri TK, Hall GS, et al. Daptomycin-resistant Enterococcus faecium in a patient with acute myeloid leukaemia. Mayo Clin Proc 2005;80(9):1215-6. 